\title{
SOCIOCULTURAL INFORMATION URBAN SPACE CONSTRUCTION
}

\author{
Olga Kononova ${ }^{1}$, Dmitry Prokudin ${ }^{1,2}$, Vitalina Karachay ${ }^{1}$ and Daria Pilysova ${ }^{1}$ \\ ${ }^{I}$ St. Petersburg National Research University of Information Technologies, Mechanics and Optics (ITMO University) \\ 197101, St. Petersburg, Russia \\ ${ }^{2}$ St. Petersburg State University 199034, St. Petersburg, Russia
}

\begin{abstract}
The construction of information urban space allows effectively improve the management quality and the sociocultural development of cities. In this paper the concept of Sociocultural Information Urban Space considered as logical development both Smart city infrastructure and social machine concepts, proposed for studying and understanding the features, possibilities, base information flows of the smart city sociocultural face. The Sociocultural Information Urban Space idea helps to recognize or improve the ordinary data driving city concept because of smart and gamification technology use so that greater range of digital services related to the sociocultural city facets can be provided to different stakeholders. Suggested smart city architecture, models, as well as St. Petersburg cases illustrate the approach, structure, and core implementation steps of the urban space. Two application scenarios allow discussing how processes of sustainable development and self-regulation mechanisms of Sociocultural Information Urban Space can be used to meet the factual needs of citizens and decision-making.
\end{abstract}

\section{KEYWORDS}

Smart City Architecture, Sociocultural Information Urban Space, Social Machine, Smart Technology, Gamification

\section{INTRODUCTION}

Modern urbanization process and urbanism have been basically conditioned by technological changes and by the organization of the city and society as a media-sphere (Wakabayashi, 2002). In this regard, the issue of description and feature characteristics determination of sociocultural information space and the study of motivation mechanisms identification for citizens' participation in the decision-making processes in urban development domain among the proposed intellectual smart cities solutions become relevant.

Generally, the concept of the Sociocultural Information Urban Space can be briefly identified as the complex of the information flows and sources of urban and Internet data that are peculiar to sociocultural urban space, including such elements as data aggregation, using the methods of search and explication of contextual knowledge, the life situations description, web services design, providing the intellectual support for socially oriented solutions, and implementation of the gamification technologies at the stage of service design. In regard to citizens, these services perform some specific functions. Providing the feedback by means of these information resources and services, citizens might be involved in the process of the sociocultural environment formation; therefore, they can actively influence this space.

Based on the analysis of academic resources on the issue of Sociocultural Information Urban Space, it is reasonable to note, that there is a lack of researches, concerning the approaches to the use of technologies of the intellectual support of socially oriented solutions in the context of the social and cultural space of the city. Moreover, it can be stated that there is the absence of the complete deterministic concept of sociocultural service oriented information space of the modern city, describing its information resources, information flows, and information needs of the population. Big data, reflecting the various aspects of the social activity, demonstrates a great potential to the support of the socially-oriented decisions adoption, however, the practice of its use is still limited by single pilot projects and is still far from a unified standard.

Thereby, the research problem, which the current study is aiming to solve, consists in the development of the methodology of intellectual support of socially oriented solutions of sociocultural service oriented 
information environment of the modern city. It is proposed to apply the complex approach to the use of intellectual/smart technologies and gamification technologies, taking into account the cross-functional nature and complex multi-layered structure of sociocultural information urban environment. It should be noted that the relevance and scientific significance of the research problem is determined by the lack of a deterministic concept of sociocultural service oriented information urban space, describing its information resources, information flows, and information needs of the population, as well as complex approaches to monitoring, collection, and analysis, clustering and aggregation of data from heterogeneous information flows, generated in the media saturated dynamically changing sociocultural information space of the modern city. Moreover, the scientific novelty of the research problem is to offer a methodology for intellectual support of socially oriented solutions of sociocultural service oriented information space of the modern city, including the design of models, methods and services, and the description of life situations and the creation of sustainable channels of interaction and orientation in urban space.

The methodology provides a complex use of gamification- and smart technologies, urban information resources, and new sources of big data that reflect different aspects of social activity (social networks data, citizens' petitions, traffic flows, economic activity of citizens, mobile applications, etc.), Internet texts and media materials, produced by individual Internet users and Internet communities in order to develop the cognitive, educational, creative interests, the formation of sociocultural needs of the urban community, providing these needs with appropriate web services, increasing the accessibility of the city and its sociocultural space for visitors, promoting the cultural interpenetration.

\section{RELATED WORKS}

It should be noted that the progress in information and communication technologies (ICT) solutions enables not only the public and private sector, but also offers the potential for the information space of sociocultural urban environment. In this regard, the essential research issues are increasing the citizen participation via tailored efficient service provision and democracy instruments, and economic development with smart joining sustainability as a key factor of the dynamic modern city promotion. In urban planning and development sphere, the ICT concept refers to the set of urban infrastructures, architectures, applications, systems, and computational and data analytics capabilities (Bibri, 2018). Many attempts to conceptualize the information space in sociocultural urban area have been made. A considerable number of investigations have been conducted in this domain. In particular, the information environment can be viewed as the intercultural communication space. Although the availability of modern information technologies by themselves does not facilitate the intercultural communication, however, it promotes mutual recognition among peoples and their cultures (Drotianko and Yahodzinskyi, 2017).

Technical (or resource) approach to the information environment defines it via the information, stored and circulating within this environment. Whereas the sociocultural concepts of the information environment refers to the space of social communications, and identifies it as an integral part of the culture. According to the Belon et al. (2015), sociocultural environment is a complex, dynamic construct that encompasses the community's social and cultural context that shapes beliefs, values, and practices, which, in turn, may foster or inhibit individual efforts to participate in physical activity. Besides, in the field of Human Computer Interaction (HCI), the sociocultural information space can be considered focusing on studying the interactions and the relationships between humans and computers (Sharma and Mehta, 2016). Masucci et al. describes the information space as the environment where information is handled in order to navigate through the city. Despite that, wider applications of these smart ICTs are indispensable to make infrastructure systems more interdependent and interconnected (Masucci et al., 2014). In regard to the cultural heritage domain, the exchanged and produced data can be opportunely exploited by a set of applications and services in order to transform a static space into a smart environment (Chianese and Piccialli, 2016). In this case, environments defined as intelligent and pervasive identify places where there is a wide spread use of embedded devices, sensors, mobile applications and services. Such an environment enables the creation of a dynamic ecosystem where a large amount of data is constantly produced and captured. Thereby, there is the necessity of relevant mechanism of data and service delivery. The problem of evaluation and care of cultural heritage through smart city enabling technologies examine in the research of Castiglione et al. (2018). The authors note that the corresponding information systems for cultural environments should possess the following technical 
features: capability to gather information from distributed and heterogeneous data sources (Social Media Networks, Digital Libraries and Archives, Multimedia Collections, Web Data Services, etc.); advanced data management techniques and technologies; ability to provide useful and personalized data to users based on their preferences and context; advanced information retrieval facilities, data analytics and other utilities/services. Despite the number of advanced digital participatory platforms (DPPs) in the information space, there are some issues and challenges that local governments face in fostering online and offline citizen engagement (Falco and Kleinhans, 2018). The authors consider the DPPs as a specific kind of collaborative social media. They argue that there are contextual, technological, and organizational factors influencing the citizen engagement. Depending on the context, they can act as challenges, making the citizen engagement limited or ineffective. Thus, the most important factor for innovative communication is a genuine understanding (at the management level) of the benefits that could be gained through DPPs and social media in general.

Based on the issue that the general goal of smart cities is to improve sustainability with help of technologies, Ahvenniemi et al. (2017) recommend use the term "smart sustainable cities" instead of "smart cities". Authors argue that as urban sustainability frameworks contain a large number of indicators measuring environmental sustainability, smart city frameworks lack environmental indicators while highlighting social and economic aspects. However, the current large gap between smart city and sustainable city frameworks suggests that there is a need for developing smart city frameworks further or re-defining the smart city concept. Digital urban spaces have the following features: to automate the process of data acquisition, storage, organization and analysis with the help of the Internet of Things, cloud computing and other technologies; to show the city's real-time performance from different perspectives; to dynamically select and integrate smart data for providing intelligent urban information services. Digital urban spaces can comprehensively integrate data resources of urban physical, social and cyber spaces to form an urban data service system, covering the entire process of data collection, organization, analysis and management (Yaxue Ma et al., 2018).

Exploring the issue of the Sociocultural Information Urban Space within the framework of smart city concepts, which consider the smart city or smart infrastructure initiatives, launched by the governments to improve the quality of citizens' life and help city managers/planners optimize the operation and management of urban infrastructures (Thomas et al., 2017). However, it should be noted that most of the concepts do not consider the Sociocultural Information Urban Space as a point of application of efforts and technologies and do not include the methods and information models for citizen engagement in its formation. Moreover, the interests and needs in involvement into dynamic activity on use and social and cultural city environment development of the categories of "temporary" citizens (labor migrants, tourists, etc.) are not considered. Another significant research problem, recognized by scientific community, but is not studied enough in academic studies, is the lack of mechanisms to motivate the citizens' participation in the decision-making processes in urban development sphere among the proposed intellectual smart cities solutions, thus, there is the absence of maximal satisfaction of the urban community needs in quality services.

Therefore, the conducted research is aimed at filling indicated gaps by preparation and theoretical justification of the concept of sociocultural service oriented information space of the modern city as an interactive form of communication between the subjects of urban space, focused on the supplementation of existing forms and types within the framework of traditional social and cultural institutions (school, club, museum, university, theater, library etc.), on the development of intellectual and creative activities of people, based on their interactions, both in real and virtual space. The preparation of the concept includes the identification of the stakeholders and analysis of their needs, and of the basic information flows and sources of urban data. Moreover, the study can be considered as a source of knowledge and facilitates the better urban design, making it more functioning and more sustainable; optimize the use of limited resources and make better decisions in Sociocultural Information Urban Space.

\section{THE CONCEPT AND THE ARCHITECTURE}

Sustainable cities face many challenges that can be alleviated through the construction of smart cities. Smart city is a set of technical solutions and organizational activities aimed at achieving the highest possible quality of the urban management. Smart technologies reveal wide opportunities for the development of the urban 
environment, which becomes smarter when the infrastructure of the city's ICTs providing most of the city's functional needs. Smart city can be related to data-driven cities (DDC) which characterized by the ability of city authorities to use data collection, processing and analysis technologies to improve the social, economic, environmental situation and enhance the living standards of residents. DDC core components are urban data in all its diversity, data processing technologies, decision-making systems. Key stakeholders, government, business, and city residents are sources and recipients of data. The balance of stakeholders' views enhances the legitimacy, support, and effectiveness of projects smart city. However, there is another smart city component, the sociocultural, which also is important for its formation and development. Modern smart city focusses not only on certain technology packages, such city is a way to form a new society; the city solving sociocultural problems, which limit the life of the urban community, define a breakthrough into the future.

The global experience of creating information systems in various spheres of human activity, recorded in the methods of the world largest consulting companies, says that any initiative to create a smart city (building from scratch or transforming an existing model) should be accompanied by a set of basic documents and materials. The smart city architecture occupies important place (Robinson, 2012; Narmeen et al., 2015). The author's metamodel of the Smart City architecture based on the Open Group TOGAF 9.2 standard is demonstrated in Figure 1.

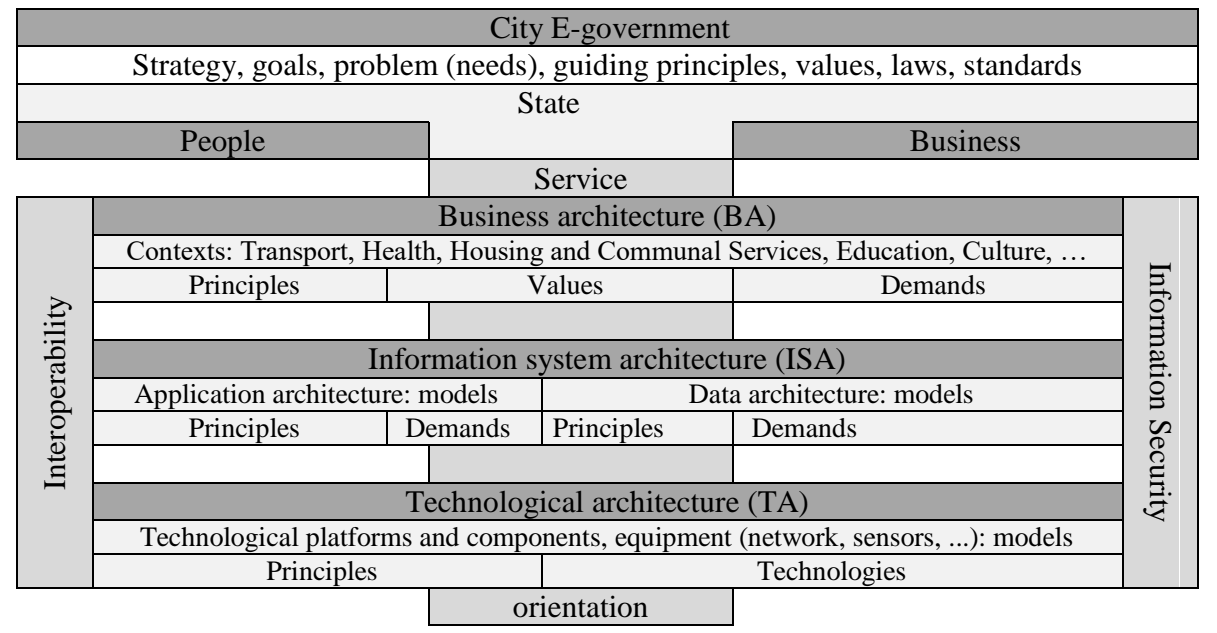

Figure 1. Metamodel of Smart City Architecture

The architecture metamodel takes a city-wide perspective and focuses on city contexts and architectural elements of every domain (Kartman et al., 2015). The metamodel helps to build the logical bridge between the definitions 'Smart City' and 'Information Urban Space' (IUS). There are several base common term-concepts for such mental migration that define every city domain content: 'Information Process', 'Application', 'Information resources', 'Big Data', 'Smart Technology'. The composition of space contexts depends on IUS type (set of urban spheres of activity). According to the authors' opinion, the Sociocultural Information Urban Space contains three base contexts, presented in Figure 2.

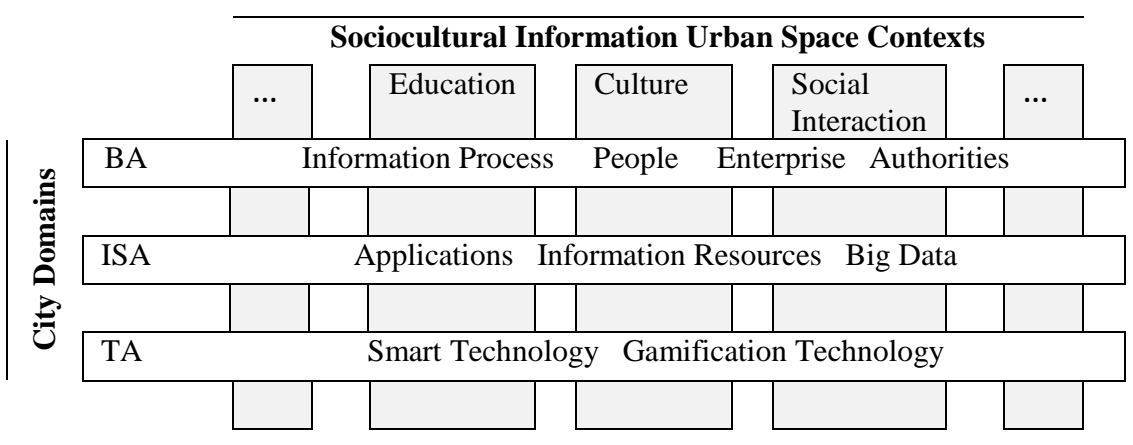

Figure 2. Domains and Contexts of Sociocultural Information Urban Space 
With the growing importance of independent social interactions gamification technologies become an important public motivation mechanism in e-government and city management, as well as self-organization, self-development one (Feng et al., 2018; Mueller et al., 2018; Vanolo et al., 2018; Zica et al., 2018; Hassan et al., 2019).

There are many IUS definitions. None of the concepts gives a complete picture of the complexity and features of the Sociocultural Information Urban Space allowed to form structure and precise description of the space. The most constructive approach took into account social aspect. The Concept of digital urban space, proposed by Chinese researchers (Ma et al., 2018), based on the conceptions and technologies of CPSS, Digital Twin, and data science proposes to use urban big data sensed and acquired from urban Cyber- Physical- Social spaces by transformation into smart data to meet the needs of urban management and operation. This approach is a kind of pragmatic and utility, however, does not sufficiently take into account the concept of social machine, corresponding with sociocultural aspects of city realities, social communications, and initiatives, sophisticate city cultural connections and interactions because of the authors seek exclusively to further activate the value and realize the use of urban data. In order to clarify the understanding of the structure and composition of the Sociocultural Information Urban Space, the authors have reviewed and compared two initial concepts associated with Smart City: information system, social machine. Information system is a system, with the associated organizational resources (human, technical, financial, and others), that provides and distributes information (ISO/IEC 2382-1:1993). In a sociotechnical perspective, information systems are composed by four components: task, people, structure (O'Hara et al., 1999).

A social machine is an environment comprising humans and technology interacting and producing outputs or action, which would not be possible without both parties present (Berners-Lee and Fischetti, 1999). Researchers (Shadbolt et al., 2019) regard social machines are co-created by their human participants and their technological components. The associative term 'co-creation' in context of social machines means - there is no simple dividing line between the human and the technological, and from the point of view of the social machine it may be irrelevant whether a particular function is performed by a person or a computer. Social machines bring people together, using technology to enable communication and cooperation at scale (Shadbolt et al., 2019). It should be noted those researchers, who consider games and gamification as examples of social machines, as the players, possibly using avatars or alternative identities, interact both with each other and with programmed machine features and AI in a synthetic environment (Castronova, 2005). The common features of the concepts presented are obvious. The authors' point of view on the development of the conceptual base is reflected in Figure 3.

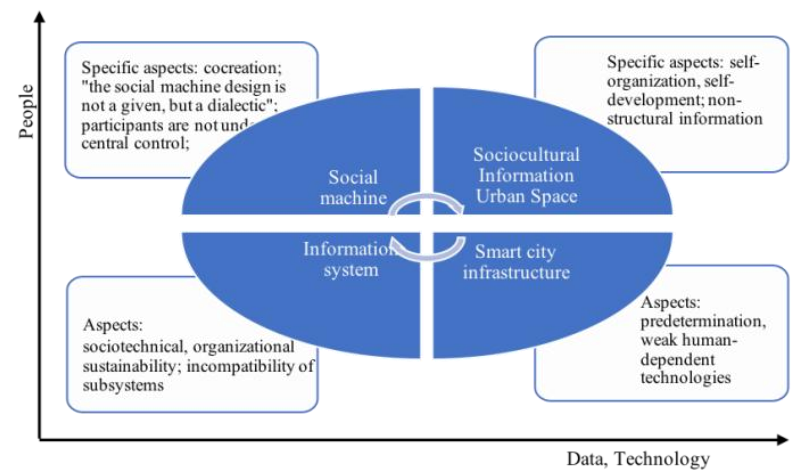

Figure 3. Sociocultural Information Urban Space Development

So, Sociocultural Information Urban Space is an evolutionary development of concepts presented with a focus on the socio-cultural development of the city. It has unique feature - the ability to self-organization and self-development, the most important for the smart city sustainability. Therefore, it is necessary to investigate and design such a space, using two scenarios at once: top-down design (purposeful planning view — city authorities, government structures, business) and bottom-up design (entropy view - personal initiatives).

Based on the construction of city profile's ideas, generalized and outlined by Ma et al. (2018), it is possible to propose specific approach to the Sociocultural Information Urban Space formation, represented as one of the components of Smart Living (Giffinger et al., 2010). There are following main components of the 
Sociocultural Information Urban Space in the electronic network environment: Internet sites; service-oriented Internet portals; social network. Besides, the components are divided into several main levels: administrative; organization-oriented level; commercial; socially oriented level. There are the different information flows between the components at different levels, which define vertical links between the levels (Figure 4). On the one hand, they are determined by the power and business impact on the person (from up to bottom): regulations, cultural policy, control (level 1); citizens involvement into the Sociocultural IUS, provision of services (levels 2, 3, 4); formation of cultural needs and its satisfaction, formation of demand (levels 3, 4). On the other hand, people themselves actively influence the formation of Sociocultural IUS in accordance with their spiritual world and individual and collective needs (from the bottom up): collective communication in social networks, cultural events discussion, individual and collective preferences formation (level 4); impact on the upper levels for the development of the Sociocultural Information Urban Space through the feedback mechanisms; impact on the cultural policy formation (levels 1-4); correction of business supply through the financial flows while services purchasing (level 3).

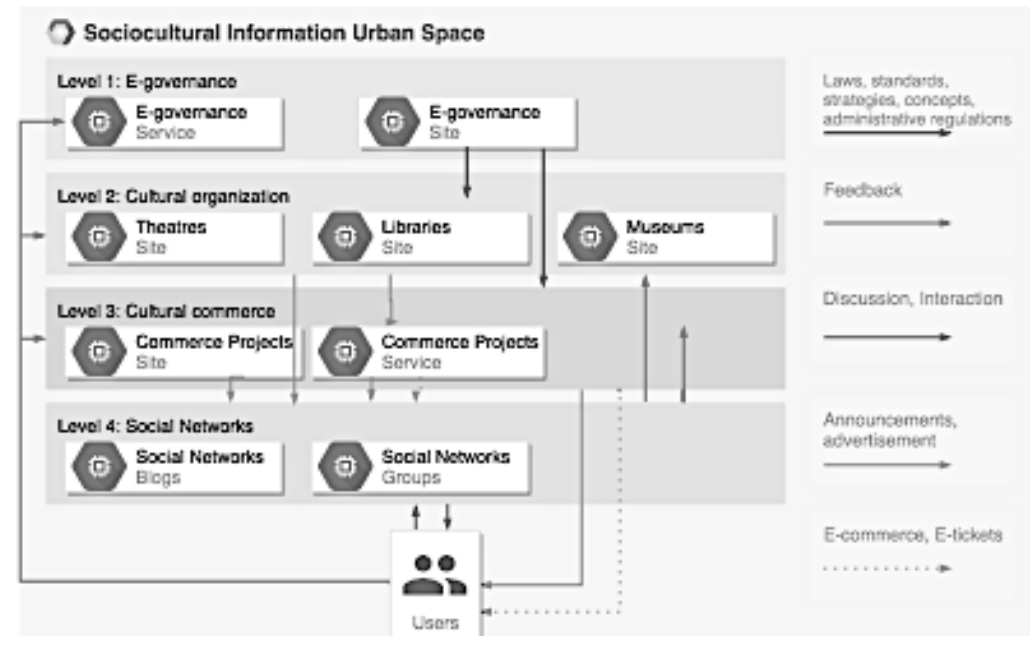

Figure 4. Architecture of Sociocultural Information Urban Space

It is obvious that the development processes of the Sociocultural Information Urban Space are possible only with a comprehensive account of all the information flows. At the same time, the city residents' involvement in active interaction within the Sociocultural Information Urban Space is the determining development factor, because the urban environment exists for the citizens and it cannot develop without their participation. In this situation, the study of information flows and its cultural contexts is a necessary condition for the cultural urban space analysis and for the operational decision-making at the level of state and municipal government. In turn, the information flows monitoring and the analysis of its contexts is a powerful analytical tool for business, which can affect the operational adjustment of the commercial offer for the cultural needs of citizens. The technique allows forming thematic collections of materials (contexts, which are relevant to the research topics). A thematic collection is a result of the peer review of paragraphs obtained sequentially with the relative, and then with thematic queries.

\section{ST. PETERSBURG CASE}

St. Petersburg (the cultural capital of Russia) have been chosen for studying the cultural information urban space. It contains all the diversity of Russian culture and the development of information infrastructure allows to identify the main components of the Sociocultural Information Urban Space. Various network information resources, which represent the different levels of the developed architecture, were considered in accordance with the approach to the formation of the Sociocultural Information Urban Space, proposed in this study. The interconnected components and the defined information flows between them were revealed by means of expert method. Special attention was paid to the gamification implementation in that components. Besides, the gamification function and the connection of information and real cultural space were also 
determined. To illustrate the pilot study, some examples are given below. Based on the analysis of the relationships between the components of the given examples, the formation of a sustainable developing Sociocultural Information Urban Space, which is one of the elements of Smart City, is concluded.

For example, several local information spaces were investigated: Anna Akhmatova Museum in the Fountain House (level 2); Museum of the history of Kronshtadt (http://visitkronshtadt.ru/) and commercial projects for the sale of tickets for cultural, sports and other events in Russia Afisha (level 3, https://www.afisha.ru). All the considered examples are characterized by the related components at both its levels, as well as at level 4 . There are information flows between the levels that implement similar functions. Next, consider the implementation of the information space of Anna Akhmatova Museum in the Fountain House (level 2). The Museum has a website (https://akhmatova.spb.ru). It performs several functions: information (Museum activities, schedule, exhibitions, announcements of events, etc); educational (additional materials, description of projects and programs for different ages); motivational (by means of the theatre project "Theatre + Museum» and gaming programs for children); feedback (guest book). Using the aggregator (https://radario.ru/hosts/4994) Anna Akhmatova Museum distributes the information about the events, sells the e-tickets for events. Besides, there is Museum group on the social network Vkontakte (https://vk.com/akhmatova.museum), which includes more than 13000 participants. The Museum publishes in the group the announcements of events and the additional cultural information, related to the theme of the Museum's exhibitions, which are discussed by the group members. There are also various documents, poems by Anna Akhmatova and by the related with Akhmatova poets, photos and videos of past events. This creates a media space of the Museum and performs a motivating function for attraction the visitors. The other thematic groups, related with this one, are the following: Information and analytical portal Brodsky.online (https://vk.com/brodsky.online) - 231 participants; Anna Akhmatova (https://vk.com/ahmatova_akuma) - more than 72000 participants; "The American Cabinet of Joseph Brodsky" (https://vk.com/brodsky.kabinet) - 1421 participants; Museum-Apartment of Lev Nikolaevich Gumilev (https://vk.com/lngumilevmuseum) - 842 participants; Project "Museum + Theatre in the Fountain House" (https://vk.com/museumplustheater) - more than 4000 participants. These groups constitute a unified information and cultural space, engaging the people in the dialogue, as well as involving the participants into its real space.

\section{CONCLUSION}

The proposed approach to the formation and study of sociocultural IUS allows concluding the following items: sustainable development of IUS is possible with maximum consideration of information flows: the sustainability is provided by information flows " from above "(formed by public and municipal authorities, cultural institutions and business); development is provided by information flows "from below" (people' initiatives and needs expressed in their activity in the Sociocultural Information Urban Space); creative activity (Social Machines) and initiatives in sociocultural IUS should be supported by citizens presence and activity at all levels of architecture; for active residents' involvement in constructive and creative activity it is necessary to use various motivational mechanisms, particularly gamification.

The study also revealed a number of prospective interdisciplinary research areas at the intersection of sociocultural IUS and gamification (including the interdisciplinary research area "Social and ethical aspects of the Digital Economy: gamification in the social, scientific and educational subject domains"), and expanded the terminological landscape. Further study development involves the development of methods for information flows monitoring between the levels and components of the Sociocultural Information Urban Space and its contexts extraction.

\section{ACKNOWLEDGEMENT}

The reported study is funded by RFBR according to the research project "The integrated approach elaboration to the analysis of the terminological base of the developing interdisciplinary research in distributed network environment” № 18-011-00923, 2018-2020. 


\section{REFERENCES}

Ahvenniemi, H. et al, 2017. What are the Differences Between Sustainable and Smart Cities? In Cities, Vol. 60, pp. 234-245. DOI: 10.1016/j.cities.2016.09.009

Belon, A. P. et al, 2015. Community Lenses Revealing the Role of Sociocultural Environment on Physical Activity. In Am J Health Promot. Vol. 30, No. 3, pp. e92-e100. DOI: 10.4278/ajhp.140428-QUAL-169

Berners-Lee, T. and Fischetti, M., 1999. Weaving the Web: The Original Design and Ultimate Destiny of the World Wide Web by its inventor. Britain: Orion Business. ISBN 978-0-7528-2090-3.

Bibri, S. E., 2018. A Foundational Framework for Smart Sustainable City Development: Theoretical, Disciplinary, and Discursive Dimensions and Their Synergies. In Sustainable Cities and Society, Vol. 38, pp. 758-794. DOI: $10.1016 /$ j.scs.2017.12.032

Castiglione, A. et al, 2018. CHIS: A Big Data Infrastructure to Manage Digital Cultural Items. In Future Generation Computer Systems, Vol. 86, pp. 1134-1145. DOI: 10.1016/j.future.2017.04.006

Chianese, A. and Piccialli, F., 2016. A Smart System to Manage the Context Evolution in the Cultural Heritage Domain. In Computers and Electrical Engineering, Vol. 55, pp. 27-38. DOI: 10.1016/j.compeleceng.2016.02.008

Drotianko, L. and Yahodzinskyi, S., 2017. Information Environment as the Intercultural Communication Space. In MATEC Web Conf., Vol. 106. 01006. DOI: 10.1051/matecconf/201710601006

Falco, E. and Kleinhans R., 2018. Beyond Technology: Identifying Local Government Challenges for Using Digital Platforms for Citizen Engagement. In International Journal of Information Management, Vol. 40, pp. 17-20. DOI: 10.1016/j.ijinfomgt.2018.01.007

Feng, Y. et al., 2018. Gamification artifacts and crowdsourcing participation: Examining the mediating role of intrinsic motivations. In Computers in Human Behavior, Vol. 81, pp. 124-136. DOI: 10.1016/j.chb.2017.12.018

Giffinger, R. and Gudrun, H., 2010. Smart cities ranking: an effective instrument for the positioning of the cities? In ACE: Architecture City \& Environment, Vol. 6, No. 12, pp. 7-26. DOI: 10.5821/ace.v4i12.2483

Hassan, L. and Hamari, J., 2019. Gamification of E-Participation: A Literature Review. Proceedings of 52nd Hawaii International Conference on System Sciences (HICSS'52), At Maui HI, USA, pp. 3077-3086.

Castronova E., 2005. Synthetic worlds: the business and culture of online games. University of Chicago Press, Chicago.

Kartman, G, Sandnes, A., Smit, G., 2011. Creating municipal ICT architectures. A reference guide from smart cities. https://joinup.ec.europa.eu/document/eu-creating-municipal-ict-architectures-reference-guide-smart-cities.

Ma, Y. et al., 2018. City profile: using smart data to create digital urban spaces. ISPRS Ann. Photogramm. Remote Sens. Spatial Inf. Sci., Vol. IV-4/W7, pp. 75-82. DOI:10.5194/isprs-annals-IV-4-W7-75-2018

Masucci, P. A. et al, 2014. Exploring the Evolution of London's Street Network in the Information Space: A Dual Approach. In Phys. Rev. E, Vol. 89, No. 1, 012805. DOI: 10.1103/PhysRevE.89.012805

Mueller, C., Klein, U., Hof, A., 2015. An easy-to-use spatial simulation for urban planning in smaller municipalities. Computers. In Environment and Urban Systems, Vol. 71, pp. 109-119 DOI: 10.1016/j.compenvurbsys.2018.05.002

Narmeen, Z. B. and Jawwad, A. S., 2015. Smart City Architecture: Vision and Challenges. In International Journal of Advanced Computer Science and Applications, Vol. 6, No. 11, pp. 246-255. DOI: 10.14569/IJACSA.2015.061132

O'Hara, M., Watson, R., Cavan, B., 1999. Managing the three levels of change. In Information Systems Management. Vol. 16, No. 3, pp. 63-70. DOI:10.1201/1078/43197.16.3.19990601/31317.9

Sharma, S. and Mehta, V., 2016. Socio-Cultural Context of Human Computer Interaction (HCI) in Online Shopping Environment. In Apeejay-Journal of Management Sciences and Technology, Vol. 4, No.1, pp. 26-36. URL: https://apeejay.edu/aitsm/journal/docs/issue-oct-2016/ajmst040103.pdf

Shadbolt, N., O'Hara, K., De Roure, D., Hall, W., 2019. The Theory and Practice of Social Machines. Springer Nature Switzerland AG, Switzerland. DOI: 10.1007/978-3-030-10889-2

Thomas, S. et al, 2017. A Master Data Management Solution to Unlock the Value of Big Infrastructure Data for Smart, Sustainable and Resilient City Planning. Creative Construction Conference 2017, CCC 2017, 19-22 June 2017, Primosten, Croatia. Procedia Engineering, Vol. 196., pp. 939-947.

Wakabayashi, M., 2002. Urban Space and Cyberspace: Urban Environment in the Age of Media and Information Technology. In International Journal of Japanese Sociology. Vol. 11, No. 1, pp. 6-18. DOI: 10.1111/14756781.00014

Vanolo, A., 2018. Cities and the politics of Gamification. In Cities, Vol. 74, pp. 320-326. DOI: $10.1016 /$ j.cities.2017.12.021

Zica, M. R., Ionica, A. C., Leba, M., 2017. Gamification in the context of smart cities. International Conference on Applied Sciences (ICAS2017), 10-12 May 2017, Hunedoara, Romania. Materials Science and Engineering. International Conference on Applied Sciences, Vol. 294, 012045. DOI: 10.1088/1757-899X/294/1/012045 